\title{
Controlling domain wall oscillations in bent cylindrical magnetic wires
}

\author{
R. Cacilhas ๑, C. I. L. de Araujo ๑, and V. L. Carvalho-Santos $\odot$ \\ Departamento de Física, Universidade Federal de Viçosa, Avenida Peter Henry Rolfs s/n, 36570-000 Viçosa, Minas Gerais, Brazil \\ R. Moreno $\odot$ \\ Earth and Planetary Science, School of Geosciences, University of Edinburgh, Edinburgh EH9 3FE, United Kingdom \\ O. Chubykalo-Fesenko \\ Instituto de Ciencia de Materiales de Madrid, CSIC, Cantoblanco, 28049 Madrid, Spain \\ D. Altbir \\ Departamento de Física, CEDENNA, Universidad de Santiago de Chile, Avenida Ecuador 3493, Santiago, Chile
}

(Received 14 November 2019; revised manuscript received 14 March 2020; accepted 27 April 2020;

published 18 May 2020)

\begin{abstract}
Magnetic cylindrical nanowires are promising candidates for future three-dimensional nanotechnology. Domain walls (DWs) in magnetic nanowires play the role of information carriers, and the development of applications requires proper description of their dynamics. Here we perform a detailed analytical and numerical analysis of the DW motion along a bent magnetic nanowire under the action of tangential magnetic fields. Our results show that the DW velocity, precession, and oscillation frequencies can be controlled by the interplay between the curvature and the external magnetic field. Small magnetic fields induce a DW motion without precession and oscillatory behavior, while higher magnetic fields yield a Walker breakdown regime, in which an oscillatory forward and backward DW motion is observed. Controlled DW motion under the Walker breakdown regime makes magnetic nanowires potential candidates for nanoscale microwave generation and sensing.
\end{abstract}

DOI: 10.1103/PhysRevB.101.184418

\section{INTRODUCTION}

Magnetic cylindrical nanowires (NWs) hold important promises for future three-dimensional (3D) nanotechnologies such as the nanoscale Internet of Things [1-3], a 3D complex platform of multiphysics interconnected devices [4] with different functionalities for information processing, communication, and sensing. The importance of magnetic nanowires for these technologies comes from the fact that they can respond to many external stimuli such as electrical, magnetic, thermal, and stress excitations. Consequently, by changing their composition or geometries, they can be functionalized for different components in such hypothetical devices. A large number of possibilities opens the door for the use of magnetic nanowires as different components of nanoscale devices. In this line, we propose that bent cylindrical magnetic nanowires can be engineered for nanoscale communication technologies, similar to spin-torque nano-oscillators [5-9]. This statement is based on the fact that when a domain wall moves under the action of a magnetic field or electric current in these nanowires, it oscillates around and along the wire axis [10-12], and the frequency can be controlled by the nanowire geometry and external magnetic field.

In magnetic nanowires, domain walls (DWs) play the role of information carriers. Depending on material parameters and geometrical constraints of the nanowire, the DW can be of the transverse or vortex (Bloch point) type [13-15]. Here we will work with the first type, which appear in cylindrical nanowires of diameters $\lesssim 20 \mathrm{~nm}$ and relatively low saturation magnetization such as in permalloy (FeNi).

The cylindrical geometry of nanowires plays an important role. First and importantly, for technological applications, it has been predicted that domain walls in straight cylindrical nanowires do not suffer from the so-called Walker breakdown phenomenon with a sudden drop in velocity due to internal instabilities, which happens in magnetic stripes [16-18]. Indeed, Yan et al. [19] showed that the DW motion along a straight magnetic nanowire with a cylindrical cross section does not present the oscillation associated with the Walker breakdown and the DW velocity varies linearly as a function of the magnetic field even for high magnetic fields. Furthermore, the changes in the geometry of magnetic nanostructures lead to curvature-induced Dzyaloshinskii-Moriya interactions (DMIs) [20,21], and thus, cylindrical nanowires are intrinsically magnetochiral systems [22]. Indeed, due to this curvature-induced DMI, intriguing geometrical effects were reported [23-27]. In the case of magnetic nanowires, it was shown that this effective DMI yields a DW pinning at the maximum of the curvature [28], and the average velocity of the DW increases as a function of the curvature [12].

Unfortunately, the presence of bends in cylindrical nanowires introduces back the Walker breakdown for the DW motion [12]. This fact, however, may be useful since, at quite low applied fields, the DW performs an oscillatory motion 


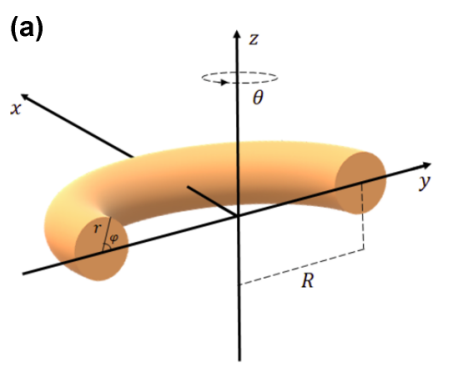

(b)

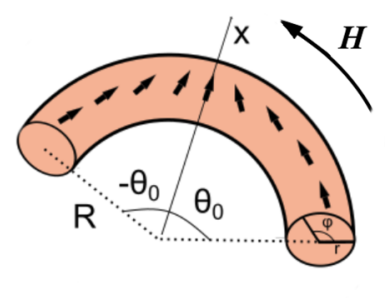

FIG. 1. (a) Sketch of the adopted coordinate system. (b) The DW configuration in a bent nanowire and the magnetic field direction.

with large amplitude along the nanowire, together with the precession around its axis. Similar behavior was described in a theoretical analysis of the DW motion along a helical nanowire induced by an electrical current [29]. This fact, to our mind, opens the possibility to generate an alternating magnetic field for microwave generation and sensing communications at the nanoscale. Since the oscillatory motion appears after the Walker breakdown, in this work, we look for its appearance. Namely, we consider the geometry of the azimuthal magnetic field in bent cylindrical nanowires, in analogy to straight nanostripes, where the field acts along the nanowire axis. Experimentally, this field can be generated by an electric current passing through the center of curvature of the nanowire.

Due to the above-described promises, the study of the influence of the curvature on the DW motion in bent cylindrical nanowires becomes very relevant. Therefore, in this work, we perform a detailed analysis of the DW dynamics along bent nanowires described as toroidal sections. Through analytical and numerical calculations, we investigate the DW motion in terms of position, phase, and velocity in bent nanowires with different curvatures and under magnetic fields. The main results suggest that the periodic motion of the DW, characterizing a Walker regime, appears when the magnetic field intensity is above a small critical value. This critical value of the magnetic field depends on the curvature of the nanowire. Above the Walker field, the oscillatory motion of the DW, which can be controlled by external parameters, appears.

\section{THEORETICAL MODEL}

In our model, we consider a bent cylindrical nanowire, described as a toroidal section with length $L$, internal and external radii $R$ and $r$, respectively, and toroidal and poloidal angles denoted by $\theta$ and $\varphi$ (see Fig. 1). In this frame, the relation among the wire length, external radius, and the toroidal angle is given by $L=2 \theta_{0} R$. Therefore, $R=L / 2 \theta_{0}$, and then a straight wire can be obtained in the limit $\theta_{0} \rightarrow 0$, while the largest curvature, described as a half-torus section, is given by $\theta_{0}=\pi / 2$. For further analysis, we also define the NW curvature as $K=R^{-1}$.

The dynamics of a DW displacing along the bent wire will be described by the Landau-Lifshitz-Gilbert (LLG) equation

$$
\frac{\partial \mathbf{m}}{\partial \tau}=\mathbf{m} \times \frac{\delta \epsilon}{\delta \mathbf{m}}+\alpha \mathbf{m} \times \frac{\partial \mathbf{m}}{\partial \tau},
$$

where $\mathbf{m}=\mathbf{M} / M_{s}$ is the normalized magnetization vector field, $M_{s}$ is the saturation magnetization, and $\tau=\left(\gamma_{0} M_{s}\right) t$ and $\epsilon=E /\left(\mu_{0} M_{s}^{2}\right)$ are the dimensionless time and normalized energy, respectively. $\mu_{0}$ is the magnetic permeability, $\alpha$ is the dimensionless Gilbert damping parameter, and $\gamma_{0}=\mu_{0}|\gamma|$ is the gyromagnetic factor $\left(\gamma=-1.76 \times 10^{11} \mathrm{rad} / \mathrm{Ts}\right)$. The magnetization $\mathbf{m}$ will be parametrized in terms of spherical coordinates lying on a curvilinear basis in the form of $\mathbf{m}=\cos \Theta \boldsymbol{e}_{\theta}+\sin \Theta \cos \Phi \boldsymbol{e}_{r}+\sin \Theta \sin \Phi \boldsymbol{e}_{z}$, where the directional vectors $\left(\boldsymbol{e}_{r}, \boldsymbol{e}_{\theta}, \boldsymbol{e}_{z}\right)$ are related to the toroidal coordinate system presented in Fig. 1. This parametrization in terms of spherical angles allows us to rewrite the LLG equation as

$$
-\sin \Theta \frac{\partial \Theta}{\partial \tau}=\frac{\delta \epsilon}{\delta \Phi}+\alpha \sin ^{2} \Theta \frac{\partial \Phi}{\partial \tau}
$$

and

$$
\sin \Theta \frac{\partial \Phi}{\partial \tau}=\frac{\delta \epsilon}{\delta \Theta}+\alpha \frac{\partial \Theta}{\partial \tau} .
$$

Aiming to obtain the energetics and dynamics of a DW displacing along a nanowire, we propose that the magnetization profile of a head-to-head DW can be described by an ansatz using the collective-variables approach [12], in the form

$$
\Theta(\theta, \tau)=2 \arctan \left[\exp \left(\frac{R \theta-q(\tau)}{\Delta}\right)\right], \quad \Phi(\tau)=\phi(\tau),
$$

where $q$ and $\phi$ represent the canonical conjugated pair of collective variables, i.e., the position and phase of the DW center. Additionally, $\Delta$ determines the DW width, which will be assumed to be constant and independent of the DW phase. Indeed, we have performed micromagnetic simulations to calculate the DW width as a function of $\phi$, and we have observed that despite the DW width being a slave variable of the DW phase $[\Delta \equiv \Delta(\phi)]$ and the curvature inducing a dependence of $\Delta$ on $\phi$, this change is less than $3 \%$. Moreover, although the magnetic field strength can change the DW width, performed micromagnetic simulations have shown that this change does not produce significant qualitative or quantitative changes in the DW dynamics, as also observed in previous works [28-30]. In this context, the approximation of a rigid domain wall is valid, and a generalized DW model [31], in which the DW width can change, will produce small differences compared to our results. Additionally, it is worth noting that we are interested in describing the DW dynamics when it displaces far from the wire borders. Therefore, because $\Delta \ll L$, near the wire ends, the magnetization configuration consists of a quasitangential configuration, and therefore, $\cos \Theta \approx \pm 1$ at $\theta= \pm \theta_{0}$. From these assumptions, the dynamics of the DW can be obtained by substituting the ansatz (4) in Eqs. (2) and (3), leading to

$$
\dot{q}=\frac{1}{2 S} \frac{\partial \epsilon}{\partial \phi}+\alpha \Delta \dot{\phi}
$$

and

$$
\dot{\phi}=-\frac{1}{2 S} \frac{\partial \epsilon}{\partial q}-\frac{\alpha}{\Delta} \dot{q},
$$

where the dot represents the derivative in relation to $\tau, S=$ $\pi r^{2}$ is the area of the circular cross section of the wire, and $\epsilon$ 
is the DW energy. This energy can be obtained by adding the three contributions to the magnetic energy, that is,

$$
\epsilon=S R \int_{-\theta_{0}}^{\theta_{0}}\left[\xi_{x}+\xi_{d}+\xi_{z}\right] d \theta,
$$

where $\xi_{x}, \xi_{d}$, and $\xi_{z}$ are, respectively, exchange, dipolar, and Zeeman energy densities.

The exchange energy density normalized to $M_{s}^{2}$ is formally defined as $\xi_{x}=\ell^{2}(\nabla \mathbf{m})^{2}$, where $\ell=\sqrt{A /\left(\mu_{0} M_{s}^{2}\right)}, A$ is the exchange stiffness, and the gradient operator is given in terms of the curvilinear basis. In this context, the exchange energy density can be written as

$$
\xi_{x}=\frac{\ell^{2}}{R^{2}}\left[\left(\frac{\partial \Theta}{\partial \theta}+\cos \Phi\right)^{2}+\left(\sin \Theta \frac{\partial \Phi}{\partial \theta}-\cos \Theta \sin \Phi\right)^{2}\right] .
$$

The dipolar energy density can be written as an easytangential anisotropy term, in the form

$$
\xi_{d}=-\lambda \cos ^{2} \Theta,
$$

where $\lambda>0$ represents an easy-tangential anisotropy constant coming from magnetostatic contributions. It is worth noting that since we are considering thin magnetic wires $(r \ll \ell, R, L)$, the dependence of $\lambda$ on $\theta$ and $\phi$ can be neglected, and then, the demagnetizing factor of the bent wire can be given by the same value calculated for a cylindrical nanowire, i.e., $\lambda=1 / 4$ [32-34].

Finally, since we are considering that the applied magnetic field is pointing along the azimuthal direction, that is, $\mathbf{H}=H \boldsymbol{e}_{\theta}$, the Zeeman energy density is given by

$$
\xi_{z}=-\frac{\ell^{2}}{A} M_{s} H .
$$

\section{RESULTS AND DISCUSSION}

The integration of the three terms accounting for the total energy density can be the performed, and the results for exchange and dipolar interactions were previously obtained (see Eqs. (A1) and (A2) of Ref. [12]). Additionally, the Zeeman energy is evaluated as

$$
E_{z}=-H S\left[L-\Delta \ln \left(\frac{1+\mathrm{e}^{\frac{-2 q+L}{\Delta}}}{1+\mathrm{e}^{\frac{-2 q-L}{\Delta}}}\right)\right] .
$$

The analysis of the energy terms reveals that only the exchange energy presents a dependence on the DW phase in such a way that the minimum exchange energy is obtained for $\phi=\pi$, and its maximum value is obtained for $\phi=0$. This result evidences the existence of a curvature-induced DW phase selection [12,27,28]; that is, a head-to-head DW has minimum energy when it is pointing out of the bent.

In our calculations we consider a permalloy wire whose exchange constant is $A=1.3 \times 10^{-11} \mathrm{~J} / \mathrm{m}, \mu_{0} M_{s}=1 T, \Delta=$ $30 \mathrm{~nm}$ (a typical value for a permalloy nanowire with a diameter of $20 \mathrm{~nm}$ ), and we fix the damping constant as $\alpha=0.01$. Simplified analytical expressions can be obtained for a bent nanowire with a small curvature, neglecting the terms of the order of $K^{2}$ in the energy calculations. In this context, we will assume that $\Delta \ll L$ and that the DW is displacing far from the border of the nanowire, that is, $q \lesssim L / 10$. In this case, we obtained simplified expressions for energy contributions, i.e.,

$$
\begin{gathered}
E_{x} \approx 2 \pi \ell^{2} S K \cos \phi, \\
E_{d} \approx-\lambda V,
\end{gathered}
$$

and

$$
E_{z} \approx-2 H S q .
$$

In this case Eqs. (5) and (6) can be simplified to

$$
\dot{\phi}=a+b \sin \phi
$$

and

$$
\dot{q}=\alpha \Delta a-\frac{\Delta}{\alpha} b \sin \phi,
$$

where $a=H /\left(1+\alpha^{2}\right)$ and $b=\alpha \pi \ell^{2} K /\left[\Delta\left(1+\alpha^{2}\right)\right]$. The above equations form a dynamical system. It is clear that there are no stationary points except in the case $a=0$, i.e., in the absence of external field. Importantly, Eq. (15) can be easily integrated to get solutions

$$
\begin{aligned}
& \phi_{1}=2 \arctan \left[-\frac{b}{a}-\frac{\sqrt{a^{2}-b^{2}}}{a} \cot \left(\frac{\sqrt{a^{2}-b^{2}}}{2} \gamma t\right)\right], \\
& \phi_{2}=2 \arctan \left[-\frac{b}{a}-\frac{\sqrt{b^{2}-a^{2}}}{a} \operatorname{coth}\left(\frac{\sqrt{b^{2}-a^{2}}}{2} \gamma t\right)\right] .
\end{aligned}
$$

In the above equations, $\phi_{1}$ and $\phi_{2}$ are the solutions for the cases $a>b$ and $a<b$, respectively. The DW position can be promptly obtained by substituting the above solutions in Eq. (16) and performing the integration in $t$. Nevertheless, the obtained equation is cumbersome, and it will be omitted here. The analysis of Eqs. (17) reveals that for $a>b$, the DW presents a precessional motion with frequency $\omega=$ $\sqrt{a^{2}-b^{2}} / 2$. Therefore, the frequency of the DW precession depends on both the magnetic field and curvature. That is, diminishing curvature induces an increase in the frequency of the precession motion of the DW. On the other hand, if $a<$ $b$, the DW phase asymptotically goes to $\phi_{0}=\arcsin (-a / b)$ and $q=\Delta a\left(1+\alpha^{2}\right) t / \alpha$, and then due to the interplay between curvature and magnetic field, the DW moves along the nanowire without precession, and its velocity is linearly proportional to the magnetic field strength. In this context, the main aspects of the behavior of the DW phase and position can be observed in Fig. 2, where we present the DW phase and position as a function of time for the curvature $K=$ $5 \times 10^{-3} \mathrm{~m}^{-1}$ and for different magnetic fields. The analysis of Fig. 2(a) reveals the dependence of the DW precession frequency on the magnetic field. That is, higher magnetic fields induce precessional motions with higher frequencies. This behavior is evidenced in the DW translational motion, presented in Fig. 2(b), where the interplay between torques induced by curvature and magnetic field produce a nonmonotonic behavior for the DW velocity. The critical field value between the translational and oscillatory regimes is defined by $H_{c r}=\alpha \pi \ell^{2} K / \Delta$. This finding is very interesting because in the regime of small curvatures there is a Walker field linearly 

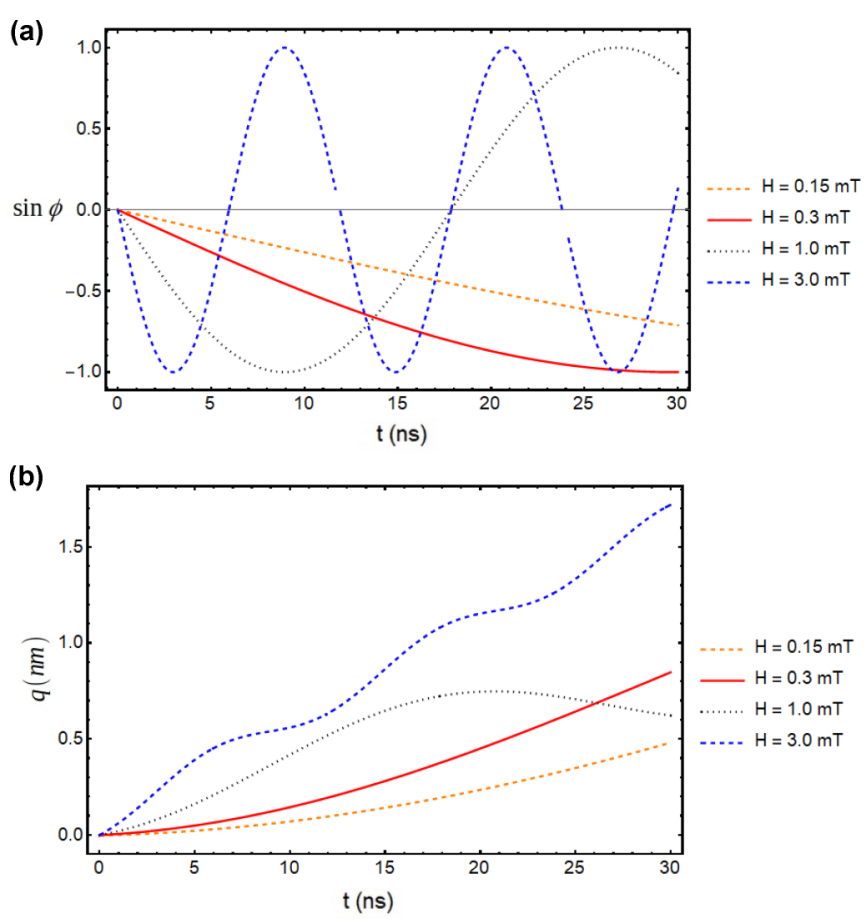

FIG. 2. (a) The DW phase as a function of the time for different magnetic fields. (b) The DW position for the same values of the magnetic field. In both plots, we have adopted $K=5 \times 10^{-3} \mathrm{~m}^{-1}$.

proportional to the curvature. This result can be promptly compared with current-induced motion of DWs in helicoidal nanowires. Indeed, in Ref. [29] the authors show that in the limit of small curvatures, there is a critical current for which the Walker breakdown occurs. The velocity associated with this critical current density is $u_{c} \propto \alpha K$. The appearance of the Walker limit when the DW propagates along a bent wire is associated with curvature-induced anisotropy [35], which induces a preferential direction to the DW phase, generating a curvature-induced phase selection for the DW [28]. Finally, for $b=0$ (corresponding to a straight wire), Eq. (17) is simplified to $\phi=\gamma a t$, according to the expected DW rotation when it is displacing along a straight wire [19].

Next, we perform a complete analysis of the DW position and phase as a function of the nanowire curvature and magnetic field based on Eqs. (5) and (6). We start by analyzing a nanowire of $L=1000 \mathrm{~nm}$ with two curvatures, $K=\pi \times 10^{6} \mathrm{~m}^{-1}$ (corresponding to $\phi_{0}=\pi / 2$ ) and $K=$ $\pi / 6 \times 10^{6} \mathrm{~m}^{-1}\left(\phi_{0}=\pi / 12\right)$, subject to an external azimuthal magnetic field of $H=3 \mathrm{mT}$. The main results are presented in Fig. 3, where we notice that in the two analyzed cases, the DW motion occurs in the Walker regime, that is, presents an oscillatory motion along the wire length. The oscillations of both DW position and phase occurring for higher curvatures lead to larger average DW velocity. Regarding results for a constant (linear) magnetic field published in Ref. [12], we can assert that higher DW velocities are obtained when applying an Oersted field.

The influence of curvature in the DW dynamics for bent nanowires is shown in Fig. 4, where the DW position under the action of a 2-mT azimuthal magnetic field is represented. Specifically, we study three different curvatures: $2 \pi \times 10^{6} \mathrm{~m}^{-1}$
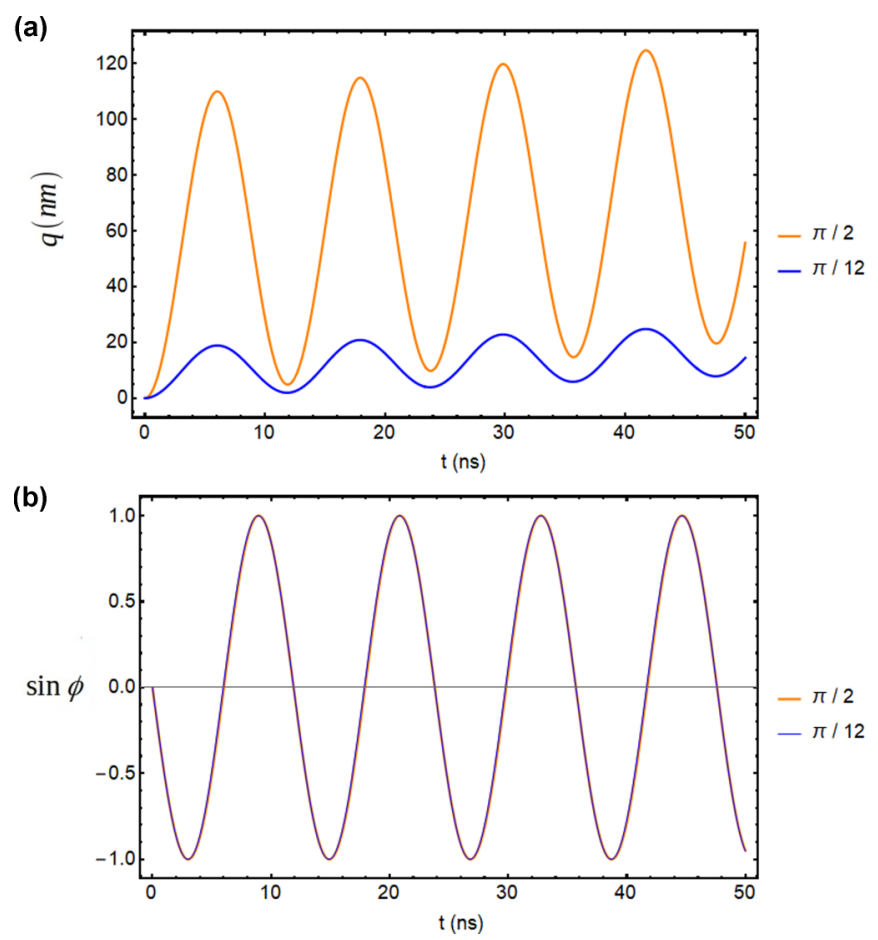

FIG. 3. (a) The DW position and (b) phase as a function of time under an azimuthal magnetic field of $3 \mathrm{mT}$ for nanowire of $L=$ $1000 \mathrm{~nm}$ with two curvature values, $K=\pi \times 10^{6} \mathrm{~m}^{-1}\left(\phi_{0}=\pi / 2\right)$ and $K=\pi / 6 \times 10^{6} \mathrm{~m}^{-1}\left(\phi_{0}=\pi / 12\right)$.

[Figs. 4(a) and 4(d)], $\pi \times 10^{6} \mathrm{~m}^{-1}$ [Figs. 4(b) and 4(e)], and $5 \pi \times 10^{5} \mathrm{~m}^{-1}$ [Figs. 4(c) and 4(f)]. Under the condition of a half-torus geometry, $L=\pi / K$, the lengths corresponding to each case are $L=500 \mathrm{~nm}, L=1 \mu \mathrm{m}$, and $L=2 \mu \mathrm{m}$, respectively, which must be taken into account to understand when the domain wall exits from the end of the nanowire. Results clearly confirm that the higher the curvature is, the higher the DW average velocity is. Furthermore, an exciting result from Fig. 4 is the possibility of controlling DW oscillations along the nanowire axis in terms of curvature. Due to the increase in the exchange energy when the DW is pointing along the internal region of the bent, there is a fast dynamics in its phase in order to bring it back to $\phi=\pi$ (pointing outward the bent), resulting in DW oscillating behavior around NW. The analysis of Figs. 4(d)-4(f) reveals that the DW phase dynamics has a small dependence on the wire length (and, consequently, on its curvature). Indeed, it can be observed that in all cases, after $5 \mathrm{~ns}$, the DW phase is $\approx-\pi / 2$, and thus, the curvature has a small influence on the DW phase precession period. Finally, one can observe that for the nanowire with $L=500 \mathrm{~nm}$ (curvature $2 \pi \times 10^{6} \mathrm{~m}^{-1}$ ), the DW does not present the oscillatory behavior observed in the other two cases. This result occurs because, in this case, the DW did not have sufficient time to change its phase from zero to $\pi$ before the arrival at the nanowire border.

The influence of the magnetic field strength on the DW motion is presented in Fig. 5 for a nanowire with length $L=2000$ $\mathrm{nm}\left(K=5 \pi \times 10^{5} \mathrm{~m}^{-1}\right)$. Again, starting from $H=1.5 \mathrm{mT}$ both DW position and phase oscillate. It can be observed that the frequency of the DW precession strongly depends 

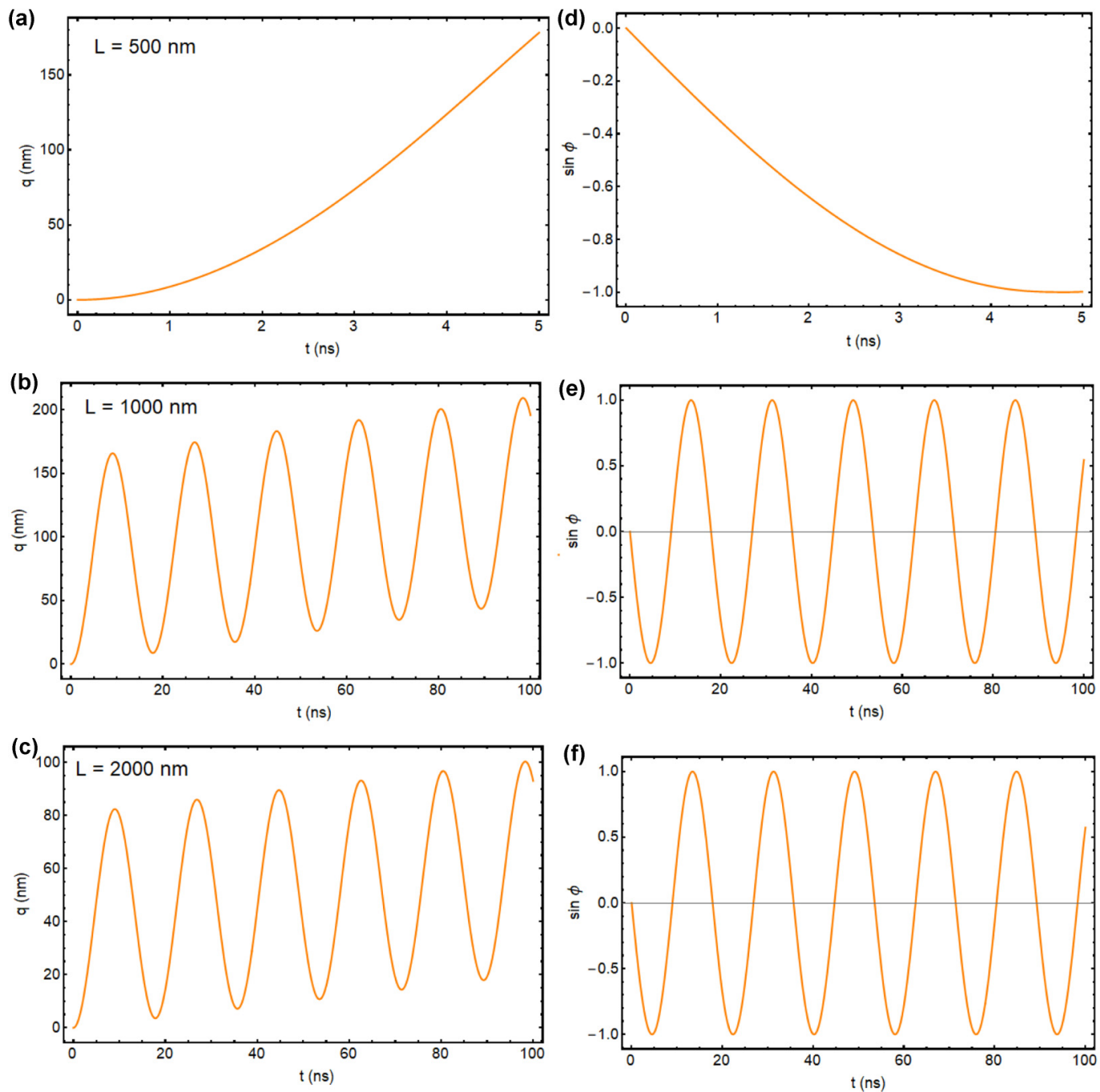

FIG. 4. The DW position as a function of time for nanowires with curvatures (a) $2 \pi \times 10^{6} \mathrm{~m}^{-1}$, (b) $\pi \times 10^{6} \mathrm{~m}^{-1}$, and (c) $5 \pi \times 10^{5} \mathrm{~m}^{-1}$ under the action of a magnetic field of $2 \mathrm{mT}$. The DW phase as a function of time for curvatures (d) $2 \pi \times 10^{6} \mathrm{~m}^{-1}$, (e) $\pi \times 10^{6} \mathrm{~m}^{-1}$, and (f) $5 \pi \times 10^{5} \mathrm{~m}^{-1}$.

on the magnetic field and can be controlled by small fields. Generally, stronger magnetic fields produce higher torques on the DW, and then the DW precesses faster. It can also be noticed that for magnetic fields above $1.5 \mathrm{mT}$, the smaller the magnetic field is, the faster the DW propagation is. However, by comparing the DW positions for $H=0.5 \mathrm{mT}$ and $H=$ $1.5 \mathrm{mT}$, we can observe that this dependence is the opposite; that is, the DW is faster in the latter case. This fact evidences the existence of a Walker breakdown phenomenon for the DW dynamics when it is displaced along a bent wire.

Applications using the DW motion along bent nanowires demand a complete understanding of the frequency and amplitude of the DW oscillation. Indeed, during the domain wall motion, the stray field in a fixed location in proximity to the nanostructure surface will either increase or decrease, generating an electromotive force. The stray field generated by a DW depends on the distance from the nanowire. It was shown that the stray field generated by a domain wall displacing along straight nanowires is on the order of $10^{5} \mathrm{~A} / \mathrm{m}$ and decays to approximately $1 \mathrm{~A} / \mathrm{m}$ at a distance of $150 \mathrm{~nm}$ [36]. Because the frequency of precession and oscillation of the DW propagating along a bent nanowire are the same, the previous results allow us to state that the curvature of the nanowire does not influence the oscillation frequency of the DW motion. In this context, we have analyzed the effects of the magnetic field strength and wire curvature on the amplitude of the DW oscillations and the influence of the magnetic field on the oscillation frequency. In Fig. 6(a), we show the amplitude of the DW oscillation as a function of the curvature for different values of the magnetic field. We can notice that due to the competition between the torques produced by the exchangedriven curvature-induced anisotropy and the magnetic field, the amplitude of the DW oscillatory motion depends on both. Indeed, because the torque produced by the magnetic field induces a faster precession motion, the amplitude of the DW oscillations decreases when the magnetic field increases. On the other hand, the increase in the curvature leads to an increase in the amplitude of the oscillations. This result occurs because the curvature-induced anisotropy generates an extra torque, and the domain wall displaces longer distances before performing a complete precession. For straight nanowires, the amplitude of the oscillations is zero, and the 

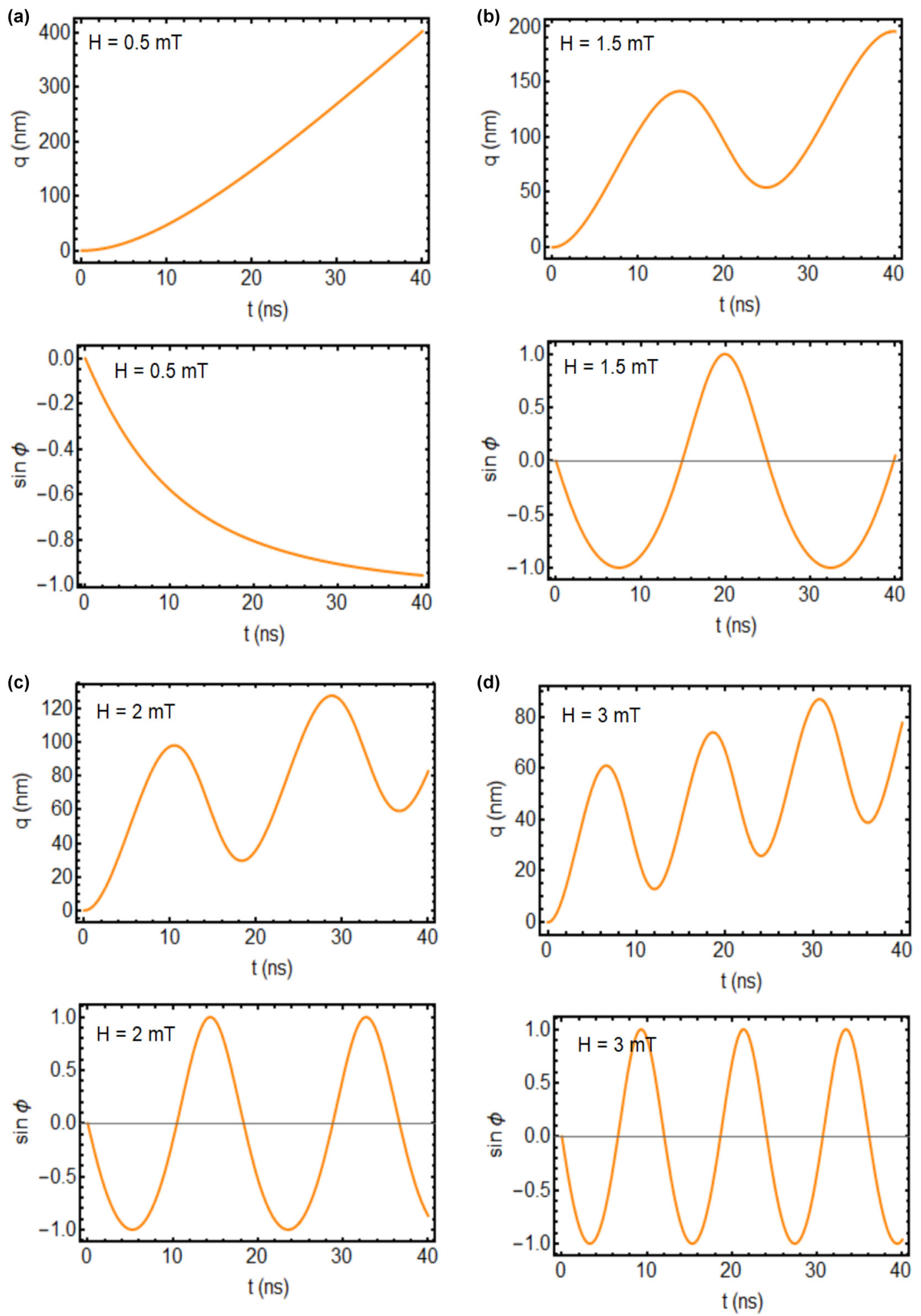

FIG. 5. (a)-(d) The DW position and phase for $\mu H=0.5,1.5,2.0$, and $3.0 \mathrm{mT}$, respectively.

DW does not present the Walker behavior during its motion, as it should be [19]. In Fig. 6(b), we show the oscillation frequency as a function of the magnetic field, where one can note that the oscillatory frequency varies linearly with the magnetic field.

We also performed calculations of the average velocity as a function of the magnetic field for different curvatures, shown in Fig. 7. The average velocity is defined as $\langle v\rangle=d / t$, where $d$ is the distance covered by the DW in $t=5 \mathrm{~ns}$, which is a proper time because, at this time, domain walls do not escape from the nanowire in any of the studied cases. The NW curvature strongly influences the average DW velocity, and more curved nanowires present larger velocities. The dependence on the magnetic field is highly nonlinear, and 


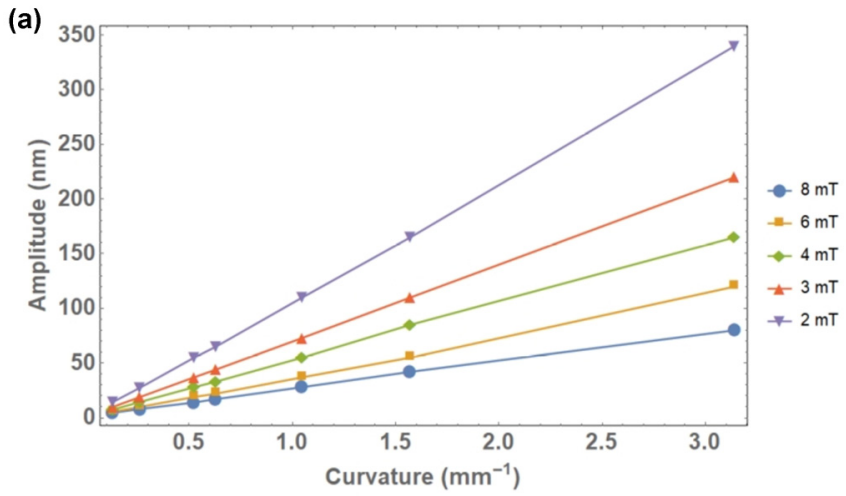

(b)

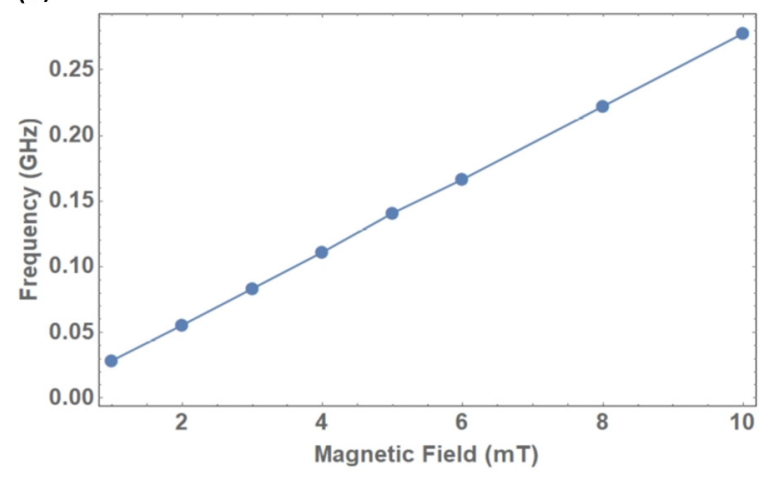

FIG. 6. (a) The influence of magnetic field and curvature on the amplitude of the oscillations of the DW propagating along a bent nanowire. (b) The behavior of the oscillation frequency as a function of the magnetic field for a nanowire with curvature $K=\pi \times 10^{6} \mathrm{~m}^{-1}$.

the presence of the Walker breakdown is evident. A small magnetic field can control its occurrence. Compared to the case of a constant nonazimuthal field considered in Ref. [12], the effect of curvature on the velocity is accentuated.

\section{CONCLUSIONS}

In conclusion, bent magnetic cylindrical nanowires can present oscillatory behavior of domain wall dynamics, controlled by an external magnetic field. While in cylindrical straight nanowires a DW rotates around the axis only by the action of a constant magnetic field, the oscillations along the axis appear in bent nanowires due to the effect of the exchange interaction. Consequently, the Walker breakdown phenomenon is recovered. With aim of determining when this situation is happening, in this paper we have analyzed the domain wall dynamics in bent wires, with a half-torus

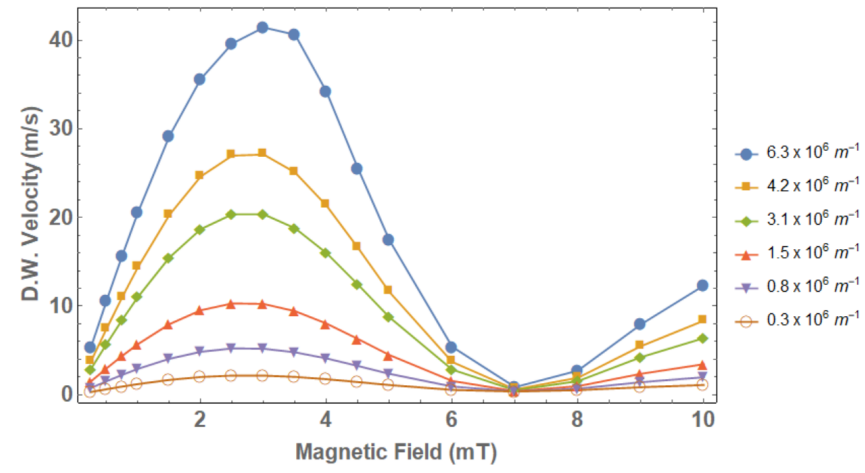

FIG. 7. DW average velocity as a function of the external magnetic field for different curvatures.

geometry, under the action of an azimuthal magnetic field. The obtained analytical results for nanowires with small curvature revealed that the oscillatory regime can appear depending on the interplay between the curvature and magnetic field.

For very small magnetic fields, the domain wall position does not present an oscillatory behavior, and it moves with velocity linearly increasing with the field. Although nanowires with larger curvatures present higher average velocities, the Walker limit appears, and a critical field value in which the DW velocity abruptly drops is observed. This drop is accompanied by the domain wall oscillations along the nanowire length, which are controlled by magnetic fields. It was also shown that the maximum DW average velocity increases as a function of the curvature in such a way that the DW velocity for a straight nanowire is recovered for small curvatures.

Overall, the results allow the control of the conditions for domain wall dynamics and, particularly, its oscillatory behavior. We believe that such control can be very useful for nanoscale communication applications such as those needed for future technologies in the Internet of Things.

\section{ACKNOWLEDGMENTS}

In Brazil, this study was financed in part by the Coordenação de Aperfeiçoamento de Pessoal de Nível Superior Brasil (CAPES) - Finance Code 001. The authors also thank CNPq (Grants No. 401132/2016-1 and No. 302084/2019-3). In Scotland, we acknowledge the Natural Environment Research Council (Grant No. NE/S011978/1). In Chile, we acknowledge financial support from FONDECYT Grant No. 1160198 and CONICYT through Centro Basal FB0807. In Spain, we acknowledge the financial support from the Ministry of Economy and Competitivity under Grant No. MAT2016-76824-C3-1-R.
[1] Y. Zhan, Y. Mei, and L. Zhengac, J. Mater. Chem. C 2, 1220 (2014).

[2] H. Sun, M. Yin, W. Wei, J. Li, H. Wang, and X. Jin, Microsyst. Technol. 24, 2853 (2018)
[3] L. Atzori, A. Iera, and G. Morabito, Comput. Networks 54, 2787 (2010).

[4] A. Fernańdez-Pacheco, R. Streubel, O. Fruchart, R. Hertel, P. Fischer, and R. P. Cowburn, Nat. Commum. 8, 15756 (2017). 
[5] J. S. Moodera, L. R. Kinder, T. M. Wong, and R. Meservey, Phys. Rev. Lett. 74, 3273 (1995).

[6] E. Bankowski, T. Meitzler, R. S. Khymyn, V. S. Tiberkevich, A. N. Slavin, and H. X. Tang, Appl. Phys. Lett. 107, 122409 (2015).

[7] M. Quinsat, F. Garcia-Sanchez, A. Jenkins, V. Tiberkevich, A. Slavin, L. Buda-Prejbeanu, A. Zeltser, J. Katine, B. Dieny, M.-C. Cyrille, and U. Ebels, Appl. Phys. Lett. 105, 152401 (2014).

[8] A. Slavin and V. Tiberkevich, Phys. Rev. Lett. 95, 237201 (2005).

[9] J. Torrejon, M. Riou, F. A. Araujo, S. Tsunegi, G. Khalsa, D. Querlioz, P. Bortolotti, V. Cros, K. Yakushiji, A. Fukushima, H. Kubota, S. Yuasa, M. D. Stiles, and J. Grollier, Nature (London) 547, 428 (2017).

[10] A. Goussev, R. G. Lund, J. M. Robbins, V. Slastikov, and C. Sonnenberg, Phys. Rev. B 88, 024425 (2013).

[11] A. Goussev, R. G. Lund, J. M. Robbins, V. Slastikov, and C. Sonnenberg, Proc. R. Soc. A 469, 20130308 (2013).

[12] R. Moreno, V. L. Carvalho-Santos, A. P. Espejo, D. Laroze, O. Chubykalo-Fesenko, and D. Altbir, Phys. Rev. B 96, 184401 (2017).

[13] H. Foster, T. Schrefl, D. Suess, W. Scholz, V. Tsianots, R. Dittrich, and J. Fidler, J. Appl. Phys. 91, 6914 (2002).

[14] A. Thiaville and Y. Nakatani, Spin Dynamics in Confined Magnetic Structures III (Springer, Berlin, 2006).

[15] S. Da Col, S. Jamet, N. Rougemaille, A. Locatelli, T. O. Mentes, B. S. Burgos, R. Afid, M. Darques, L. Cagnon, J. C. Toussaint, and O. Fruchart, Phys. Rev. B 89, 180405(R) (2014).

[16] N. L. Schryer and L. R. Walker, J. Appl. Phys. 45, 5406 (1974).

[17] T. Ono, H. Miyajima, K. Shigeto, K. Mibu, N. Hosoito, and T. Shinjo, Science 284, 468 (1999).

[18] A. Mougin, M. Cormier, J. P. Adam, P. J. Metaxas, and J. Ferré, Europhys. Lett. 78, 57007 (2007).
[19] M. Yan, A. Kákay, S. Gliga, and R. Hertel, Phys. Rev. Lett. 104, 057201 (2010).

[20] R. Hertel, SPIN 03, 1340009 (2013).

[21] Y. Gaididei, V. P. Kravchuk, and D. D. Sheka, Phys. Rev. Lett. 112, 257203 (2014).

[22] D. D. Sheka, V. P. Kravchuk, and Y. Gaididei, J. Phys. A 48, 125202 (2015).

[23] P. Landeros and A. S. Núñez, J. Appl. Phys. 108, 033917 (2010).

[24] M. Charilaou, H.-B. Braun, and J. F. Löffler, Phys. Rev. Lett. 121, 097202 (2018).

[25] J. A. Fernandez-Roldan, R. Perez del Real, C. Bran, M. Vazquez, and O. Chubykalo-Fesenko, Nanoscale 10, 5923 (2018).

[26] S. Vojkovic, V. L. Carvalho-Santos, J. M. Fonseca, and A. S. Nunez, J. Appl. Phys. 121, 113906 (2017).

[27] R. G. Elías, N. Vidal-Silva, and V. L. Carvalho-Santos, Sci. Rep. 9, 14309 (2019).

[28] K. V. Yershov, V. P. Kravchuk, D. D. Sheka, and Y. Gaididei, Phys. Rev. B 92, 104412 (2015).

[29] K. V. Yershov, V. P. Kravchuk, D. D. Sheka, and Y. Gaididei, Phys. Rev. B 93, 094418 (2016).

[30] O. V. Pylypovskyi, D. D. Sheka, V. P. Kravchuk, K. V. Yershov, D. Makarov, and Y. Gaididei, Sci. Rep. 6, 23316 (2016).

[31] V. P. Kravchuk, J. Magn. Magn. Mater. 367, 9 (2014)

[32] D. G. Porter and M. J. Donahue, J. Appl. Phys. 95, 6729 (2004).

[33] R. Moskovitz and E. D. Torre, IEEE Trans. Magn. 2, 739 (1966).

[34] V. V. Slastikov and C. Sonnenberg, IMA J. Appl. Math. 77, 220 (2012).

[35] R. Streubel, P. Fischer, F. Kronast, V. P. Kravchuk, D. D. Sheka, Y. Gaididei, O. G. Schmidt, and D. Makarov, J. Phys. D 49, 363001 (2016).

[36] D. Su, K. Wu, R. Saha, and J.-P. Wang, J. Appl. Phys. 126, 183902 (2019). 